\title{
RASSEGNA
}

\section{La sarcoidosi: vecchie certezze e nuove prospettive}

\section{Sarcoidosis: old certainties and new perspectives}

\section{Claudio Tana $^{\mathrm{a}, \mathrm{b}, *}$, Marco Tana $^{\mathrm{a}}$, Andrea Mezzetti ${ }^{\mathrm{a}}$, Cosima Schiavone ${ }^{\mathrm{a}, \mathrm{b}}$}

\author{
a Dipartimento di Medicina e Scienze dell'Invecchiamento, Ospedale Clinicizzato SS. Annunziata, \\ Università degli Studi Gabriele D’Annunzio, Chieti \\ ${ }^{\mathrm{b}}$ UO Dipartimentale di Ecografia Internistica, Ospedale Clinicizzato SS. Annunziata, \\ Università degli Studi Gabriele D’Annunzio, Chieti
}

Ricevuto il 2 aprile 2012; accettato il 9 maggio 2012 disponibile online il 23 giugno 2012

\section{PAROLE CHIAVE \\ Sarcoidosis; \\ HLA antigens; \\ Granuloma; \\ Peptidyl-dipeptidase A; Adalimumab.}

\begin{abstract}
Summary
Introduction: Sarcoidosis is the paradigm of the internistic disease. It virtually affects every organ and tissue and is characterized by the presence of noncaseating granulomas. A holistic view is needed for the diagnosis and treatment of the disease. The authors provide an overview on several recently published studies, which pose new perspectives on the approach to the disease. Materials and methods: The authors reviewed the scientific literature focusing on new etiopathogenetic, diagnostic and therapeutic data.

Results and discussion: A multimodal approach is appropriate and effective in the diagnosis and treatment of systemic diseases such as sarcoidosis. In this context, the internist plays a key role more than any other specialist.
\end{abstract}

(c) 2012 Elsevier Srl. All rights reserved.

\section{Introduzione}

La sarcoidosi è una malattia multisistemica infiammatoria cronica a eziologia sconosciuta, caratterizzata dalla formazione di granulomi in organi e tessuti affetti, e può essere causa di morbilità significativa [1]. Il termine "sarcoidosi", coniato nel 1899 dal dermatologo norvegese Caesar Boeck, deriva dalla fusione delle parole greche sark e oid, letteralmente "condizione carnosa" [2]. È nota anche come malattia di Besnier-Boeck-Schaumann o granulomatosi sistemica o reticoloendoteliosi benigna.

Colpisce principalmente soggetti di età compresa tra 25 e 40 anni [3], soprattutto di sesso femminile (rapporto femmine-maschi 2:1). Un secondo picco è stato riscontrato nelle donne di età compresa tra 45 e 65 anni. L'Europa del Nord è la regione a più alta incidenza, con circa 5-40 casi

\footnotetext{
* Corrispondenza: Dipartimento di Medicina e Scienze dell’Invecchiamento, Ospedale Clinicizzato SS. Annunziata, via dei Vestini 29 - 66100 Chieti.

E-mail: claudio.tana@yahoo.it (C. Tana).
} 
per 100.000 abitanti. Negli Stati Uniti l'incidenza è maggiore negli afroamericani (35,5/100.000 persone/anno), colpiti circa 3 volte di più rispetto alle popolazioni di razza caucasica $(10,9 / 100.000$ persone/anno) [4].

Un recente studio epidemiologico condotto in Croazia ha riportato un'incidenza media annuale pari a 3,3 persone colpite ogni 100.000 abitanti [5].

La mortalità nella sarcoidosi è maggiore nei pazienti afroamericani, nei quali la malattia è più spesso cronica e coinvolge un maggior numero di organi [6]. In base a uno studio di Swigris et al. [7], il tasso di mortalità correlato alla sarcoidosi è aumentato significativamente negli Stati Uniti tra il 1988 e il 2007, in particolare nelle donne afroamericane di età uguale o superiore a 55 anni.

In Giappone e negli Stati Uniti le principali cause di morte sono costituite, rispettivamente, dal coinvolgimento cardiaco e polmonare.

\section{Cenni eziopatogenetici}

Recenti studi hanno fornito maggiori informazioni sulle cause della sarcoidosi. Fattori di rischio occupazionali importanti sarebbero costituiti dalla lavorazione agricola, dall'esposizione a muffe e a pesticidi industriali. Nello studio Access i pazienti, rispetto ai soggetti controllo, riferivano meno frequentemente abitudini tabagiche $[8,9]$. Un'aumentata incidenza di sarcoidosi è stata riscontrata tra i "rescue workers" esposti alle macerie delle Torri Gemelle in seguito all'evento terroristico dell'11 settembre 2001 [10]. In passato sono stati proposti svariati agenti infettivi come causa della sarcoidosi. Negli ultimi anni la proteina catalasi-perossidasi (mKatG) del Mycobacterium tuberculosis è stata identificata come un potenziale antigene coinvolto nella patogenesi [11]. Gli aspetti genetici della malattia sono stati ulteriormente chiariti. Un polimorfismo a carico del gene BTNL2 (coinvolto nel processo di presentazione dell'antigene) e i geni codificanti per HLA di classe 1 e 2 potrebbero aumentare il rischio di sviluppare la patologia [8].

In base agli attuali modelli patogenetici, la malattia verrebbe scatenata dall'esposizione a un antigene peptidico non ancora ben noto, il quale verrebbe presentato alle cellule T nel contesto del complesso maggiore di istocompatibilità (fase 1). Polimorfismi genetici e alcuni HLA rispetto ad altri provocherebbero una maggiore suscettibilità individuale. Questi eventi innescherebbero la formazione di granulomi (fase 2). Successivamente la malattia potrebbe andare incontro a remissione o a cronicizzazione, con o senza fibrosi polmonare (fase 3) [2,8].

\section{Meccanismi fisiopatologici dell'ipercalcemia}

Tra le varie manifestazioni clinico-laboratoristiche della sarcoidosi è opportuno soffermarsi sui meccanismi fisiopatologici che sottendono le alterazioni del metabolismo del calcio/vitamina $D$, conducendo a ipercalcemia e ipercalciuria fino al 5-10\% e al 40-50\% dei casi, rispettivamente.

Secondo ipotesi accreditate [12], i macrofagi, contenuti nei granulomi sarcoidei e in quelli alveolari, stimolati dalla presenza di grandi quantità di interferone-gamma secrete dai linfociti Th1, produrrebbero, attraverso l'1-alfa-idrossilazione della vitamina $D$, alti livelli di 1,25-diidrossivitamina
$D_{3}\left[1,25(\mathrm{OH})_{2}-D_{3}\right]$. Essi, inoltre, attraverso la secrezione di citochine come il fattore di necrosi tumorale-alfa (TNF-alfa) e l'interleuchina-6 (IL-6), attiverebbero l'espressione del gene codificante per il peptide correlato al paratormone (PTH-rP) all'interno dei granulomi sarcoidei e il relativo processo trascrizionale. La condizione di ipercalcemia si instaurerebbe, quindi, da un lato, per un aumentato assorbimento intestinale di calcio ad opera di 1,25-diidrossivitamina $D_{3}$ e, dall'altro, per un aumentato riassorbimento osseo da parte degli osteoclasti reclutati e attivati dalla $1,25(\mathrm{OH})_{2}-\mathrm{D}_{3}$ e dal PTH-rP. L'ipercalcemia, dipendendo dal grado di attività della malattia, è usualmente transitoria nella sarcoidosi subacuta, mentre può persistere durante le riacutizzazioni di malattia. L'ipercalciuria, molto più frequente dell'ipercalcemia, vedrebbe come meccanismi fisiopatologici l'aumentato assorbimento del calcio ad opera di $1,25(\mathrm{OH})_{2}$ $\mathrm{D}_{3}$ con un alto rapporto calcio urinario/creatinina, un massivo riassorbimento osseo per estesa disseminazione di malattia e conseguenti osteopenia e osteoporosi, e, infine, la produzione del fattore attivante gli osteoclasti da parte di linfociti attivati, cellule mononucleate e granulomi.

L'ipercalcemia e l'ipercalciuria, se non adeguatamente monitorate e trattate, potrebbero portare a lungo termine all'instaurarsi di temibili complicanze come la nefrocalcinosi, l'insufficienza renale e le pancreatiti [13].

\section{Aspetti clinici}

La presentazione clinica dipende dall'estensione e della severità del coinvolgimento d'organo. Nel $5 \%$ dei casi la malattia è asintomatica e riscontrata incidentalmente a un esame radiografico del torace. Nel $45 \%$ dei casi si può manifestare con sintomi sistemici quali febbre, anoressia e artralgie. In alcuni casi l'esordio può essere acuto e caratterizzato da linfoadenopatia ilare bilaterale, eritema nodoso e artralgie (sindrome di Lofgren). Secondo lo studio ACCESS, la presentazione iniziale e le caratteristiche cliniche sarebbero strettamente correlate a fattori epidemiologici come sesso, razza ed età [14].

\section{Manifestazioni polmonari}

La sarcoidosi coinvolge più frequentemente $i$ polmoni e $i$ linfonodi intratoracici (oltre il $90 \%$ dei pazienti ha un interessamento polmonare) [15]. Nel $50 \%$ dei casi i sintomi principali sono costituiti da dispnea da sforzo, tosse, dolore toracico [2]. L'emottisi è un'evenienza rara. La malattia può colpire l'intero apparato respiratorio, ma determina più spesso deficit restrittivo ai test di funzionalità respiratoria, configurandosi come malattia interstiziale; può coinvolgere, inoltre, grandi e piccole vie aeree causando deficit ostruttivo.

Complicanze comuni sono costituite da atelettasie, fibrosi, lesioni bollose, bronchiectasie, lesioni cavitarie e micetomi [15]. La malattia può inoltre associarsi ad ipertensione polmonare, complicanza che limita gravemente lo stato funzionale, richiede più spesso la supplementazione di ossigeno e candida più frequentemente i pazienti al trapianto polmonare [16].

Raro è l'interessamento pleurico con versamenti, ispessimento, fibrosi, calcificazioni, chilotorace, pneumotorace o 
emotorace [17], o di sedi quali laringe $(0,5 \%)$, naso e seni paranasali (1\%) determinando congestione, formazione di croste, anosmia, epistassi e poliposi $[18,19]$.

\section{Possibili manifestazioni extrapolmonari}

Virtualmente la sarcoidosi può colpire qualsiasi distretto corporeo. Nella tabella 1 vengono elencati gli organi coinvolti e le caratteristiche cliniche [12,20-36]; nella tabella 2 si riportano le percentuali di interessamento delle varie sedi.

\section{Diagnosi}

La sarcoidosi è una diagnosi di esclusione meglio supportata dalla presenza dei seguenti tre elementi:

- dati clinico-radiologici compatibili;

Tabella 1 Organi coinvolti e manifestazioni cliniche della sarcoidosi descritti in letteratura.

\begin{tabular}{|c|c|}
\hline Organo & Caratteristiche cliniche \\
\hline Linfonodi periferici [20] & $\begin{array}{l}\text { Frequenti: linfoadenopatia palpabile, a consistenza dura, asintomatica, solitamente } \\
\text { cervicale, ascellare, epitrocleare e inguinale }\end{array}$ \\
\hline Occhi $[12,21]$ & $\begin{array}{l}\text { Maggiore frequenza nella popolazione giapponese con: } \\
\text { - formazione di noduli granulomatosi dell'iride, sinechie anteriori o posteriori, vasculite, } \\
\text { lesioni della coroide, aumentata pressione oculare } \\
\text { - uveite anteriore, solitamente autolimitantesi, che può manifestarsi con fotofobia, visione } \\
\text { offuscata e arrossamento oculare o essere asintomatica } \\
\text { - uveite posteriore, spesso cronica, che può associarsi a neurite ottica con cecità permanente } \\
\text { se non trattata tempestivamente } \\
\text { Proptosi uni o bilaterale per infiltrazione retrorbitaria da tessuto sarcoide }\end{array}$ \\
\hline Cute $[22,23]$ & $\begin{array}{l}\text { Noduli sottocutanei, eritema nodoso e maculopapulare soprattutto agli arti inferiori, lupus } \\
\text { pernio, alopecia }\end{array}$ \\
\hline Fegato [24-26] & $\begin{array}{l}\text { - Test di funzionalità epatica alterati (GOT,GPT, gamma-GT, fosfatasi alcalina) fino al } 24 \% \text { dei } \\
\text { casi } \\
\text { - Altri reperti: presenza di noduli epatici, epatomegalia, riscontro di cirrosi (raro), } \\
\text { ipertensione portale, rottura di varici esofagee, sindrome di Budd Chiari, insufficienza } \\
\text { epatica, carcinoma epatocellulare }\end{array}$ \\
\hline Cuore $[27,28]$ & $\begin{array}{l}\text { - Interessamento cardiaco più frequente e a prognosi più severa nella popolazione giapponese } \\
\text { rispetto a quella europea e americana, caratterizzato dalla presenza di aritmie (fibrillazione } \\
\text { atriale, blocco di branca completo, tachicardie ventricolari), disfunzione ventricolare } \\
\text { sinistra, morte improvvisa } \\
\text { - Raro il riscontro di cardiomiopatia ipertrofica }\end{array}$ \\
\hline Apparato muscolo-scheletrico [29] & $\begin{array}{l}\text { - Interessamento articolare: artralgie, poliartrite acuta, raramente deformante; sindrome di } \\
\text { Lofgren (linfoadenopatia ilare bilaterale, eritema nodoso e artralgie) } \\
\text { - Localizzazioni ossee: dattilite e osteolisi } \\
\text { - Interessamento muscolare: diffuso, forma pseudotumorale; miositi } \\
\text { - Alterazioni del metabolismo osseo: ipercalcemia spesso asintomatica, ipercalciuria }\end{array}$ \\
\hline Milza [30] & $\begin{array}{l}\text { Splenomegalia solitamente asintomatica, raramente con riduzione di conta piastrinica, } \\
\text { globuli rossi e bianchi; granulomi }\end{array}$ \\
\hline Parotidi [65] & Tumefazione ghiandolare \\
\hline $\begin{array}{l}\text { Sistema nervoso centrale } \\
\text { e periferico [31] }\end{array}$ & $\begin{array}{l}\text { Paralisi del nervo facciale, crisi epilettiche, febbricola, cefalea, ipertensione endocranica, } \\
\text { disturbi visivi, meningite asettica, neuropatia dei nervi intracranici, pseudotumor, lieve deficit } \\
\text { cognitivo, psicosi, delirium, demenza, disorientamento, amnesia, proptosi, polineuropatie } \\
\text { assonali, mononeuropatie, radicolopatie, disturbi sensoriali agli arti inferiori, emiplegia, } \\
\text { iperriflessia, disartria, disfagia, oftalmoplegia, emorragia cerebrale, ictus ischemico }\end{array}$ \\
\hline
\end{tabular}

Apparato neuroendocrino [12,31] - Lesioni sarcoidee a carico dell'asse ipotalamico-ipofisario e/o delle ghiandole periferiche: alterata regolazione della temperatura corporea, obesità patologica, sonnolenza marcata preceduta o seguita da insonnia, alterazioni della personalità, SIADH, diabete insipido, alterato controllo del senso di sete con polidipsia e poliuria in presenza di normali valori di $\mathrm{ADH}$, iperprolattinemia, deficit di $\mathrm{GH}$, ipotiroidismo e/o iposurrenalismo secondari, ipotiroidismo e/o iposurrenalismo primitivi, ipogonadismo ipogonadotropo e conseguenti amenorrea e galattorrea

- Disordini autoimmuni: morbo di Addison isolato o associato a ipotiroidismo primitivo (sindrome di Schmidt), malattia di Flaiani-Basedow-Graves 
Tabella 1 (Continua)

\begin{tabular}{ll}
\hline Organo & Caratteristiche cliniche \\
\hline Tratto gastrointestinale & Viene interessato prevalentemente lo stomaco (antro) con sintomatologia caratterizzata da \\
[32,33] & anoressia, perdita di peso, nausea, vomito per la presenza di progressivo ispessimento, \\
& rigidità delle pareti e riduzione del lume tale da mimare la presenza di un carcinoma, oppure \\
& con sanguinamenti del tratto gastrointestinale superiore per la presenza di ulcere \\
& difficilmente guaribili \\
- & Rari il coinvolgimento di piccolo intestino e colon, che può mimare il morbo di Chron, o la \\
& presenza di pancreatite acuta e masse pancreatiche \\
\hline Reni $[34]$ & Insfficienza renale cronica, nefrite tubulo-interstiziale, glomerulonefrite proliferativa \\
& extracapillare, glomerulonefrite membranosa, litiasi renale, nefrocalcinosi \\
\hline Altre sedi $[12,35,36]$ & Apparato genitale maschile: massa o noduli scrotali a consistenza duro-elastica, epididimo- \\
& orchite con dolore e gonfiore locali, oligo-azoospermia \\
- Apparato genitale femminile: lesioni a carico dell'utero con amenorrea, menorragia o \\
metrorragia, sanguinamento postmenopausale. In letteratura sono inoltre descritte lesioni \\
ovariche, a carico delle tube di Falloppio e della placenta \\
- Mammella: può simulare un carcinoma mammario. La biopsia rivela la componente \\
granulomatosa tipica
\end{tabular}

Legenda: GOT = glutammico-ossalacetico-transaminasi; GPT = glutammico-piruvico-transaminasi; gamma-GT = gamma-glutamil-transpeptidasi; $\mathrm{SIADH}=$ sindrome da inappropriata secrezione di $\mathrm{ADH} ; \mathrm{ADH}=$ ormone antidiuretico o vasopressina; $\mathrm{GH}=$ ormone della crescita.

- riscontro bioptico tessutale di granulomi a cellule epitelioidi, non caseosi;

- assenza di agenti noti (microrganismi, particelle) capaci di formare granulomi $[8,23,37]$.

Una valutazione clinica iniziale è mostrata nella tabella 3 $[23,37]$.

\section{Esami di laboratorio}

Nei pazienti con sarcoidosi l'emocromo può documentare, in caso di marcata splenomegalia, la presenza di piastrinopenia, anemia e leucopenia [30]. Rara è la pancitopenia per interessamento del midollo osseo [38]. Reperto comune è invece costituito dal riscontro di alterati valori di funzionalità epatica [24]. Come già menzionato, il riscontro di ipercalciuria è più frequente (fino al $40-50 \%$ dei casi) rispetto all'aumento dei valori di calcemia sierica (5-10\%). È opportuno, pertanto, dosare nei pazienti la calcemia sierica e il calcio urinario delle 24 ore [13,39].

\section{Biomarcatori}

È tradizionalmente noto come $\mathrm{i}$ livelli sierici dell'enzima di conversione dell'angiotensina (sACE), prodotto dai granulomi, possano aumentare nella sarcoidosi. Tale parametro è dotato, tuttavia, di scarsa sensibilità e specificità; può

Tabella 2 Interessamento percentuale dei vari distretti corporei.

Distretto corporeo

Interessamento

Riscontro clinico

Riscontro autoptico

Sistema nervoso centrale e periferico

$5-10 \%$

$25 \%$

Occhi 25-80\%

Parotidi 6\%

Linfonodi periferici 15\%

Polmoni > >90\%

Cuore

$5 \%$

Fegato

$11,5-35 \%$

$20-30 \%$

Milza

$5-10 \%$

Reni

$<1 \%$

Tratto gastrointestinale

$<1 \%$

Cute

25-35\%

Apparato genitale

Raro

Muscoli

$1,4-2,9 \%$

Articolazioni

$25-39 \%$ 
Tabella 3 Valutazione clinica iniziale.

- Anamnesi (sintomatologia, storia familiare, esposizione a fattori di rischio) ed esame obiettivo

- Radiografia del torace in due proiezioni (antero-posteriore e laterale)

- Test di funzionalità polmonare (spirometria con broncodilatatore, capacità polmonare totale, DLCO)

- Elettrocardiogramma

- Valutazione oculistica (lampada a fessura, esame tonometrico e fondoscopico)

- Emocromo completo, calcemia sierica, creatinina, fosfatasi alcalina, GOT, GPT

- Dosaggio ACE sierico (dati controversi)

- Esame bioptico di organi affetti

- Test alla tubercolina

Altri esami in base agli organi coinvolti:

- Cuore: elettrocardiogramma dinamico secondo Holter, ecocardiogramma, tomografia a emissione di positroni, risonanza magnetica, studi elettrofisiologici

- Polmone: cateterismo cardiaco destro (ipertensione polmonare)

- Sistema nervoso centrale: risonanza magnetica con gadolinio e analisi del liquido cerebrospinale

Legenda: DLCO = test di diffusione del monossido di carbonio; GOT = glutammico-ossalacetico-transaminasi; GPT = glutammico-piruvicotransaminasi; $\mathrm{ACE}=$ enzima di conversione dell'angiotensina.

risultare, infatti, normale in caso di malattia cronica e/o terapia immunosoppressiva [2,23]. La concentrazione sierica di ACE, inoltre, varia in base alla presenza di differenti polimorfismi genetici. Nei soggetti di razza caucasica, infatti, la delezione (D) o inserzione (I) dell'introne 16 sul cromosoma 17 (che codifica normalmente l'ACE), può determinare rispettivamente aumento o riduzione dell'ACE sierico, con maggiori concentrazioni nel genotipo $D / D$ e minori in quello I/I. Pertanto, sarebbe opportuno utilizzare range di riferimento differenti a seconda dei vari polimorfismi citati [40].

Un recente studio di Biller et al. [41] ha suggerito un possibile ruolo protettivo, nei confronti della sarcoidosi, della presenza contemporanea di genotipi in eterozigosi $D / /$ e $A / C$; questi dati, però, non sono confermati da Kruit et al. [42]. L'utilizzo di sACE nella diagnosi e nel monitoraggio della malattia è, pertanto, controverso [23].

Negli ultimi anni sono stati valutati numerosi biomarker in grado di predire l'attività infiammatoria della malattia. Tra questi, i livelli sierici iniziali del recettore solubile dell'interleuchina 2 (sIL2-R), del lisozima e di una glicoproteina ad alto peso molecolare espressa sugli pneumociti alveolari tipo II e sulle cellule epiteliali bronchiali (KL-6) potrebbero riflettere la presenza di alveolite linfocitaria nella malattia a localizzazione polmonare [43]. I valori di sIL-2R risulterebbero infatti aumentati nella sarcoidosi in fase acuta (tempo dalla diagnosi $\leq 2$ anni), soprattutto nella forma polmonare non in trattamento, con alti valori di sensibilità $(82 \%)$, specificità $(94 \%)$, valore predittivo positivo $(82 \%)$ e negativo (94\%) [44]. In un recente studio di Petereit et al. [45] i livelli di sIL-2R misurati nel liquido cerebrospinale di pazienti con neurosarcoidosi non trattata, che risultavano superiori a $150 \mathrm{pg} / \mathrm{mL}$, possedevano un'accuratezza diagnostica del $93 \%$ rispetto ad altre malattie non infettive del sistema nervoso centrale.

\section{Lavaggio broncoalveolare ed esame istologico}

L'esame del lavaggio broncoalveolare (BAL) può documentare la presenza di alveolite linfocitica ( $>10 \%$ ) con elevato rapporto CD4/CD8. Questo dato, associato alla presenza di eosinofili e neutrofili nel range di normalità e all'assenza di plasmacellule, può essere d'aiuto nella diagnosi differenziale con le più comuni malattie interstiziali polmonari. Tale esame manca, tuttavia, di sensibilità e specificità adeguate e non può essere utilizzato isolatamente a fini diagnostici. De Smet et al. [46] hanno quindi proposto un algoritmo di calcolo basato sulla combinazione di SACE, linfociti e rapporto CD4/CD8 nel BAL: un risultato $\leq 0,07$ escluderebbe la diagnosi di sarcoidosi polmonare, mentre un valore $>0,81$ la renderebbe molto probabile. Valori intermedi verrebbero considerati non conclusivi (fig. 1).

Attualmente, nella maggioranza dei casi, la diagnosi è prettamente istologica (almeno 4 prelievi bioptici), attraverso la documentazione di granulomi non caseosi e l'assenza di cellularità e aspetti tipici per altre malattie quali linfomi e tubercolosi. La diagnosi differenziale è di cruciale importanza; un incauto inserimento della terapia steroidea potrebbe, infatti, influenzare negativamente il decorso clinico. La biopsia non è peraltro necessaria nei casi di sindrome di Lofgren [2]. L'esame bioptico della linfoadenopatia mediastinica può essere eseguito mediante agoaspirazione transbronchiale tradizionale (TBNA) 0 guidato attraverso l'ultrasonografia endobronchiale (EBUS-TBNA); quest'ultima tecnica è da prediligere, ove disponibile, in quanto avrebbe una maggiore resa diagnostica, con valori di sensibilità e specificità, rispettivamente, dell' $83,3 \%$ e del $100 \%$ in confronto a quella tradizionale $(60,9 \%$ e $100 \%$, rispettivamente), evitando così esami diagnostici maggiormente invasivi (mediastinoscopia, biopsia a cielo aperto) [47].

\section{Esami strumentali}

Un esame radiografico del torace può documentare la presenza di linfoadenopatia ilare bilaterale, isolata (stadio I) o associata a infiltrazione parenchimale (stadio II), oppure può evidenziare la presenza di infiltrazione parenchimale senza adenopatia bilaterale (stadio III) o, infine, di fibrosi associata o meno ad aspetto a nido d'ape, retrazione ilare ed enfisema (stadio IV) [37]. La classificazione in stadi possiede, tuttavia, diversi limiti: non ha una successione sequenziale, presenta una grande variabilità interosservatore, soprattutto tra gli stadi II-III e III-IV [2] e, infine, può risultare discordante 


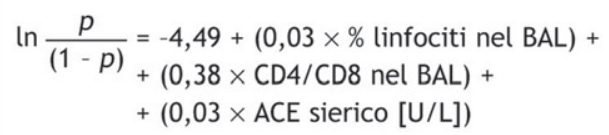

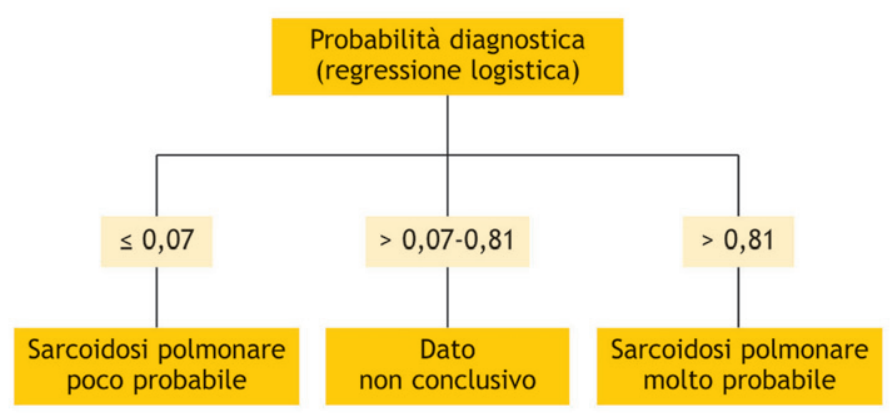

Fonte: De Smet D, et al. Am J Clin Pathol 2010;134(6):939-47.

Figura 1 Algoritmo proposto per la diagnosi di sarcoidosi polmonare.

con i test di funzionalità respiratoria nel $50 \%$ dei casi. È stato pertanto proposto di quantificare l'interessamento polmonare in base all'estensione di malattia anziché allo staging; questo correlerebbe meglio con i test di funzionalità respiratoria [48].

La tomografia computerizzata (TC) ad alta risoluzione, integrata con quella con mezzo di contrasto per lo studio del mediastino, possiede numerosi vantaggi rispetto all'esame radiografico di base, tra cui una sensibilità maggiore nel valutare il coinvolgimento linfonodale, polmonare e bronchiale. Inoltre, documentando reperti tipici (patognomonici) o atipici, può fornire informazioni preziose riguardanti l'attività della malattia e svelare segni precoci di fibrosi o di altre complicanze [49].

Altri esami (ecografia dell'addome ed eventuale completamento con TC) possono essere suggeriti dai reperti clinicolaboratoristici.

Per molti anni la scintigrafia con Gallio-67 ha rappresentato un'indagine fondamentale nel valutare il grado di attività di malattia. Recentemente, la tomografia a emissione di positroni (PET) con 18F-fluoro-desossiglucosio (FDG) ha documentato una resa diagnostica superiore, con valori di sensibilità del $97 \%$ verso l' $88 \%$ dell'esame scintigrafico, particolarmente in sedi come mediastino, linfonodi, regioni ilari ed extrapolmonari, possedendo, inoltre, una maggiore concordanza interosservatore. Si è dimostrata utile, inoltre, per individuare gli organi candidabili alla biopsia [50,51]. A causa del minor costo e della maggiore disponibilità, tuttavia, la scintigrafia con Gallio67 è ragionevolmente ancora utilizzata in caso di flare di malattia nota da tempo.

Recentemente alcuni autori hanno suggerito un approccio multimodale per meglio definire l'interessamento cardiaco: in primo luogo un ecocardiogramma, che può documentare la presenza di ispessimento e ipocinesia parietale; successivamente una risonanza magnetica con gadolinio, che può mostrare zone di iperintensità nelle sequenze T2-pesate con enhancement tardivo nella sede interessata; infine, una PET con 18F-FDG, che può mostrare uptake dalla regione di interesse, dato suggestivo di infiammazione in fase acuta [52]. Nei casi dubbi, un esame angiografico può escludere la presenza di coronaropatia quale causa di ipo-acinesia ventricolare.

\section{Principi terapeutici}

Il decorso clinico della sarcoidosi può essere progressivo nel $10-30 \%$ dei casi, conducendo a danno d'organo severo. I corticosteroidi costituiscono la terapia cardine iniziale, soprattutto nei casi di malattia severa e danno d'organo, mentre l'aggiunta di farmaci citotossici può esser utile nella gestione a lungo termine (al fine di ridurre gli effetti collaterali corticosteroideo-indotti) oppure nei soggetti refrattari alla terapia tradizionale. La durata del trattamento dovrebbe essere di almeno 12 mesi [53-55].

\section{Corticosteroidi}

È noto come la terapia cortisonica sia efficace nel sopprimere l'attività infiammatoria innescata da stimoli meccanici, chimici, infettivi e immunologici. Essa agisce principalmente inibendo geni proinfiammatori, come quelli codificanti per le citochine IL-1 e TNF-alfa o molecole di adesione, e in parte inducendo geni antinfiammatori come quelli codificanti per gli antagonisti del recettore della IL-1. In caso di coinvolgimento polmonare, vi è un'evidenza sostanziale che questi farmaci migliorino i sintomi, la funzionalità respiratoria e $i$ reperti radiografici del torace in un periodo di 6-24 mesi [56]. Le manifestazioni oculari e cutanee di entità lieve possono esser trattate con formulazioni topiche. I cortisonici inalatori vengono indicati nei pazienti con tosse cronica e coinvolgimento delle vie aeree, oppure per sospendere o ridurre al minimo $i$ glucocorticoidi orali.

Le principali indicazioni a un trattamento sistemico sono costituite dalla presenza di coinvolgimento oculare (in caso di fallimento del trattamento topico), neurologico, renale, cardiovascolare, laringeo oppure di manifestazioni polmonari severe, lupus pernio o ipercalcemia marcata $(>3,0$ $\mathrm{mmol} / \mathrm{L})$. Inizialmente i pazienti dovrebbero ricevere prednisone o prednisolone al dosaggio di 0,5-1 mg/ kg al giorno per 6-12 settimane fino a ottenere la completa remissione, seguito da una riduzione graduale della dose ogni 6-12 settimane [53]. In alcuni casi sono stati descritti fenomeni di resistenza al trattamento, verosimilmente dovuti al rilascio eccessivo di TNF-alfa dai macrofagi alveolari, motivo per cui 
tali pazienti potrebbero trarre beneficio da farmaci biologici quali gli anti-TNF-alfa (infliximab) [57].

Il principale limite all'utilizzo dei cortisonici è rappresentato dall'elevato numero di eventi avversi, in relazione agli alti dosaggi somministrati e alla lunga durata di trattamento.

\section{Immunosoppressori}

Il metotrexato è un farmaco antagonista della sintesi dell'acido folico e agisce inibendo in modo competitivo e reversibile la diidrofolato-reduttasi. Gli effetti antinfiammatori vengono mediati dall'aumento dei livelli extracellulari di adenosina. L'efficacia nei confronti della sarcoidosi è in larga parte aneddotica e basata su piccoli studi [58]. Un trial randomizzato controllato condotto da Baughman et al. [59] ha provato che i pazienti che ricevevano metotrexato necessitavano di dosaggi di corticosteroidi significativamente inferiori dopo 12 mesi rispetto al gruppo di controllo. Il metotrexato, pertanto, può essere utilizzato nei pazienti con fallimento, controindicazioni o necessità di alti dosaggi di corticosteroidi (>10 mg/die) [53].

Azatioprina, ciclofosfamide, ciclosporina e mofetil micofenolato sono stati impiegati in alcuni casi di malattia severa e/o refrattaria, ma le evidenze sono limitate [58].

\section{Antimalarici}

La clorochina e l'idrossiclorochina sono efficaci nelle localizzazioni cutanee della sarcoidosi, in caso di ipercalcemia e di coinvolgimento neurologico [37]. È preferibile l'idrossiclorochina, per il minor rischio di tossicità oculare. La dose raccomandata è di $400 \mathrm{mg} / \mathrm{die}$, anche se alcuni pazienti possono beneficiare di dosaggi inferiori $(200 \mathrm{mg} / \mathrm{die})$. Durante il trattamento è opportuno eseguire visite oculistiche ogni 6-12 mesi, per identificare eventuali segni precoci di danno retinico [2].

\section{Farmaci biologici}

Sono stati condotti vari trial clinici sugli inibitori del TNFalfa. L'etanercept si è dimostrato inefficace nel trattamento della sarcoidosi oculare in un trial randomizzato controllato e in quella polmonare in uno studio in aperto; sono stati descritti alcuni casi di sviluppo di sarcoidosi paradossa dopo somministrazione di anti-TNF-alfa, in particolar modo etanercept. In due trial randomizzati controllati con placebo, l'infliximab si è dimostrato efficace nella sarcoidosi a interessamento extrapolmonare, mentre non ha dimostrato vantaggi rispetto al placebo nel trattamento delle manifestazioni polmonari [60]. In un recente studio prospettico osservazionale condotto da Milman et al. [61] per valutare l'efficacia di adalimumab $40 \mathrm{mg}$ somministrato per via sottocutanea ogni 2 settimane in 10 pazienti con sarcoidosi attiva (dimostrata dal maggiore uptake a un esame PET con 18F-FDG), è stata documentata una riduzione della captazione in 9 pazienti (6 casi di captazione nei polmoni, 5 nei linfonodi e 4 in sedi extratoraciche). L'adalimumab non si è, tuttavia, mostrato efficace nel migliorare i test di funzionalità respiratoria, i livelli sierici di ACE e la concentrazione linfocitaria.
Alla luce di questi dati, sono necessari ulteriori trial randomizzati controllati che possano quantificare meglio il profilo di efficacia e sicurezza di tali farmaci nella sarcoidosi.

Non sono ancora disponibili dati sui farmaci modulatori delle citochine come il rituximab, il golimumab e l'ustekinumab.

\section{Follow-up}

La sarcoidosi è una malattia il più delle volte autolimitantesi, andando spesso incontro a remissione spontanea; il follow-up di una coorte di 215 pazienti valutati a distanza di 2 anni dall'arruolamento nello studio ACCESS [62] ha dimostrato che la maggioranza $(80 \%)$ presentava stabilità o miglioramento in termini di sintomatologia dispnoica, alterazioni alla radiografia del torace e indici di funzionalità respiratoria (FEV1, FVC). Una minor parte presentava, invece, un'evoluzione aggressiva con flare ripetuti. Fattori prognostici negativi erano costituiti dalla presenza di coinvolgimento extrapolmonare, basso reddito familiare annuo e razza afroamericana. Il monitoraggio risulta fondamentale: nei primi 2 anni dalla presentazione clinica $i$ pazienti dovrebbero essere valutati ogni 3-6 mesi. Dopo una completa remissione (spontanea o terapeutica) tutti i pazienti dovrebbero essere seguiti per un periodo minimo di 3 anni [2].

In passato l'ACE sierico, riflettendo la quantità totale di granulomi sarcoidei, era stato proposto come marker per valutare l'attività infiammatoria della malattia. In un lavoro recente di Miyoshi et al. [43], che ha correlato alcuni marker di attività di malattia come sACE, sIL-2R, lisozima, KL-6 e il materiale tratto dal BAL, la capacità discriminativa dell'ACE tra pazienti con e senza progressione dell'infiltrazione parenchimale è risultata molto bassa $(58,1 \%)$ con valori di specificità del 50\% [43]. Nello stesso studio il BAL, invece, documentando un aumento di cellule totali e di linfociti, ha predetto la presenza di infiltrazione parenchimale con buoni valori di sensibilità/specificità, rispettivamente del 76,9/56,7\% e del 69,2/70\%.

Un altro lavoro ha altresì dimostrato come l'8-isoprostano, un marker di perossidazione lipidica, possa costituire un fattore prognostico positivo se presente in basse concentrazioni iniziali nel materiale tratto dal BAL. Una riduzione dell'8-isoprostano in soggetti trattati non risultava, tuttavia, correlata con la regressione di malattia, riflettendo un effetto non specifico del trattamento [63].

Come già accennato, negli ultimi anni alcuni biomarker si sono dimostrati utili nel predire il grado di attività di malattia. Oltre a evidenziare come i livelli sierici iniziali di sIL-2R, lisozima e KL-6 correlino significativamente con il numero di cellule totali e linfociti CD4+ nel materiale tratto dal BAL di pazienti con sarcoidosi polmonare, Miyoshi et al. [43] hanno dimostrato che i livelli sierici iniziali di KL-6 tendono a essere significativamente associati a progressione dell'infiltrazione parenchimale alla radiografia del torace, con quadri radiologici più sfavorevoli per valori di KL-6 $\geq 354 \mathrm{U} / \mathrm{mL}$.

Altri biomarcatori come la chemochina CCL18 e la chitotriosidasi plasmatica, prodotti in grande quantità dai macrofagi sarcoidei, potrebbero risultare utili nel follow-up dei pazienti in quanto tenderebbero ad aumentare in caso di riattivazione di malattia e a ridursi per la presenza di terapia 
cortisonica. Sono tuttavia necessari ulteriori lavori prima di una loro validazione clinica [64].

\section{Conclusioni}

La sarcoidosi è una malattia spesso misconosciuta: non di rado la presentazione clinica è silente o subdola, con sintomi aspecifici. Una valutazione clinico-anamnestica accurata e un corretto uso degli esami laboratoristico-strumentali sono fattori essenziali per formulare la diagnosi. In tale ambito l'internista assume un ruolo chiave: un approccio multimodale che generi una visione olistica del paziente è prioritario in fase iniziale, mentre una gestione strettamente specialistica e focalizzata su singoli organi e apparati può essere utile ad uno step successivo.

\section{Conflitto di interessi}

Gli autori dichiarano di non aver nessun conflitto di interessi.

\section{Bibliografia}

[1] lannuzzi MC, Fontana JR. Sarcoidosis: clinical presentation, immunopathogenesis, and therapeutics. JAMA 2011;305(4): 391-9.

[2] Grutters JC, Drent M, van den Bosch JMM. Sarcoidosis. Eur Respir Mon 2009;46:126-54.

[3] Nunes H, Soler P, Valeyre D. Pulmonary sarcoidosis. Allergy 2005;60(5):565-82.

[4] Rybicki BA, Major M, Popovich Jr J, Maliarik MJ, lannuzzi MC. Racial differences in sarcoidosis incidence: a 5-year study in a health maintenance organization. Am J Epidemiol 1997;145(3): 234-41.

[5] Mise K, Jurcev-Savicevic A, Goic-Barisic I, Kozul K, Gudelj I. Sarcoidosis and tuberculosis in South Croatia: are there epidemiological similarities or not? Public Health 2011;125(10): 734-7.

[6] Israel HL, Karlin P, Menduke H, DeLisser OG. Factors affecting outcome of sarcoidosis. Influence of race, extrathoracic involvement, and initial radiologic lung lesions. Ann N Y Acad Sci 1986;465:609-18.

[7] Swigris JJ, Olson AL, Huie TJ, Fernandez-Perez ER, Solomon J, Sprunger D, et al. Sarcoidosis-related mortality in the United States from 1988 to 2007. Am J Respir Crit Care Med 2011;183(11): 1524-30.

[8] Morgenthau AS, Iannuzzi MC. Recent advances in sarcoidosis. Chest 2011;139(1):174-82.

[9] Newman LS, Rose CS, Bresnitz EA, Rossman MD, Barnard J, Frederick $M$, et al. ACCESS Research Group. A case control etiologic study of sarcoidosis: environmental and occupational risk factors. Am J Respir Crit Care Med 2004;170(12):1324-30.

[10] Jordan HT, Stellman SD, Prezant D, Teirstein A, Osahan SS, Cone JE. Sarcoidosis diagnosed after September 11, 2001, among adults exposed to the World Trade Center disaster. J Occup Environ Med 2011;53(9):966-74.

[11] Song Z, Marzilli L, Greenlee BM, Chen ES, Silver RF, Askin FB, et al. Mycobacterial catalase-peroxidase is a tissue antigen and target of the adaptive immune response in systemic sarcoidosis. J Exp Med 2005;201(5):755-67.

[12] Porter N, Beynon HL, Randeva HS. Endocrine and reproductive manifestations of sarcoidosis. QJM 2003;96(8):553-61.

[13] Myhill PC, Yeap BB. Severe hypercalcaemia in sarcoidosis with musculoskeletal involvement. Intern Med J 2009;39(3):207-9.
[14] Baughman RP, Teirstein AS, Judson MA, Rossman MD, Yeager Jr $\mathrm{H}$, Bresnitz EA, et al., Case Control Etiologic Study of Sarcoidosis (ACCESS) research group. Clinical characteristics of patients in a case control study of sarcoidosis. Am J Respir Crit Care Med 2001;164(10 Pt 1):1885-9.

[15] Morgenthau AS, Teirstein AS. Sarcoidosis of the upper and lower airways. Expert Rev Respir Med 2011;5(6):823-33.

[16] Corte TJ, Wells AU, Nicholson AG, Hansell DM, Wort SJ. Pulmonary hypertension in sarcoidosis: a review. Respirology 2011; 16(1):69-77.

[17] Armengol G, Bernet J, Lahaxe L, Lévesque H, Marie I. Uncommon manifestation revealing sarcoidosis. Rev Med Interne 2009;30(1):53-7.

[18] Reed J, deShazo RD, Houle TT, Stringer S, Wright L, Moak 3rd JS. Clinical features of sarcoid rhinosinusitis. Am J Med 2010; 123(9):856-62.

[19] Gulati S, Krossnes B, Olofsson J, Danielsen A. Sinonasal involvement in sarcoidosis: a report of seven cases and review of literature. Eur Arch Otorhinolaryngol 2012;269(3):891-6.

[20] Izzo L, Di Cello P, Meloni P, Caputo M, Izzo P, Costi U, et al. Monolateral adenopathy of the axilla due to sarcoid-type granulomata: idiopathic granulomatous disease or sarcoidosis? A case report. G Chir 2008;29(8-9):362-4.

[21] Varron L, Abad S, Kodjikian L, Sève P. Sarcoid uveitis: Diagnostic and therapeutic update. Rev Med Interne 2011;32(2):86-92.

[22] Kong F, Leng XM, Li L, Zhang FC. The clinical analysis of 35 patients with cutaneous sarcoidosis. Zhonghua Nei Ke Za Zhi 2011;50(5):397-400.

[23] lannuzzi MC, Rybicki BA, Teirstein AS. Sarcoidosis. N Engl J Med 2007;357(21):2153-65.

[24] Cremers J, Drent M, Driessen A, Nieman F, Wijnen P, Baughman $\mathrm{R}$, et al. Liver-test abnormalities in sarcoidosis. Eur J Gastroenterol Hepatol 2012;24(1):17-24.

[25] Yoshiji H, Kitagawa K, Noguchi R, Uemura M, Ikenaka Y, Aihara $Y$, et al. A histologically proven case of progressive liver sarcoidosis with variceal rupture. World J Hepatol 2011; $3(10): 271-4$.

[26] Van Brusselen D, Janssen CE, Scott C, Bevers N, Roskams T, Wouters $\mathrm{C}$, et al. Budd-Chiari syndrome as presenting symptom of hepatic sarcoidosis in a child, with recurrence after liver transplantation. Pediatr Transplant 2012;16(2):E58-62.

[27] de Ruvo E, Lanzillo C, Calò L. Arrhythmic manifestation of sarcoidosis. Europace 2011;13(8):1194.

[28] Nunes H, Freynet O, Naggara N, Soussan M, Weinman P, Diebold $B$, et al. Cardiac sarcoidosis. Semin Respir Crit Care Med 2010;31(4):428-41.

[29] Hamdi W, Néji O, Ghannouchi MM, Kaffel D, Kchir MM. Rheumatologic manifestations of sarcoidosis. Tunis Med 2010;88(11): 773-82.

[30] Moller DR. Rare manifestations of sarcoidosis. Eur Respir Monograph 2005;10:233-50.

[31] Titlic M, Bradic-Hammoud M, Miric L, Punda A. Clinical manifestations of neurosarcoidosis. Bratisl Lek Listy 2009;110(9): 576-9.

[32] Maàmouri N, Guellouz S, Ben Hariz F, Ketari S, Belkahla N, Ouerghi $\mathrm{H}$, et al. Gastrointestinal sarcoidosis. Rev Med Interne 2010;31(4):262-7.

[33] Tsintsadze MR, Beridze TM, Völker UU, Klöppel GG, Schauer RJ. Sporadic pancreatic head sarcoidosis: a rare clinical case analysis. Georgian Med News 2011;(191):26-32.

[34] Kaaroud H, Fatma LB, Beji S, Jeribi A, Maiz HB, Moussa FB, et al. Interstitial and glomerular renal involvement in sarcoidosis. Saudi J Kidney Dis Transpl 2008;19(1):67-71.

[35] Smyth LG, Long RM, Lennon G. A case of epididymal sarcoidosis. Can Urol Assoc J 2011;5(5):E90-1.

[36] Panzacchi R, Gallo C, Fois F, Dalpiaz G, Cucchi MC, Degli Esposti $\mathrm{R}$, et al. Primary sarcoidosis of the breast: case description and review of the literature. Pathologica 2010;102(3):104-7. 
[37] Statement on sarcoidosis. Joint Statement of the American Thoracic Society (ATS), the European Respiratory Society (ERS) and the World Association of Sarcoidosis and Other Granulomatous Disorders (WASOG) adopted by the ATS Board of Directors and by the ERS Executive Committee, February 1999. Am J Respir Crit Care Med 1999;160(2):736-55.

[38] Yanardağ H, Pamuk GE, Karayel T, Demirci S. Bone marrow involvement in sarcoidosis an analysis of 50 bone marrow samples Haematologia (Budap) 2002;32(4):419-25.

[39] Berliner AR, Haas M, Choi MJ. Sarcoidosis: the nephrologist's perspective. Am J Kidney Dis 2006;48(5):856-70.

[40] Biller H, Zissel G, Ruprecht B, Nauck M, Busse Grawitz A, Müller-Quernheim J. Genotype-corrected reference values for serum angiotensin-converting enzyme. Eur Respir J 2006;28(6): 1085-90.

[41] Biller H, Ruprecht B, Gaede KI, Müller-Quernheim J, Zissel G. Gene polymorphisms of ACE and the angiotensin receptor AT2R1 influence serum ACE levels in sarcoidosis. Sarcoidosis Vasc Diffuse Lung Dis 2009;26(2):139-46.

[42] Kruit A, Ruven HJ, Grutters JC, van den Bosch JM. Angiotensin II receptor type $11166 \mathrm{~A} / \mathrm{C}$ and angiotensin converting enzyme I/ D gene polymorphisms in a Dutch sarcoidosis cohort. Sarcoidosis Vasc Diffuse Lung Dis 2010;27(2):147-52.

[43] Miyoshi S, Hamada H, Kadowaki T, Hamaguchi N, Ito R, Irifune K, et al. Comparative evaluation of serum markers in pulmonary sarcoidosis. Chest 2010;137(6):1391-7.

[44] Rothkrantz-Kos S, van Dieijen-Visser MP, Mulder PG, Drent M. Potential usefulness of inflammatory markers to monitor respiratory functional impairment in sarcoidosis. Clin Chem 2003; 49(9):1510-7.

[45] Petereit HF, Reske D, Tumani H, Jarius S, Markus Leweke F, Woitalla D, et al. Soluble CSF interleukin 2 receptor as indicator of neurosarcoidosis. J Neurol 2010;257(11):1855-63.

[46] De Smet D, Martens GA, Berghe BV, Meysman M, Heylen O, Gorus FK, et al. Use of likelihood ratios improves interpretation of laboratory testing for pulmonary sarcoidosis. Am J Clin Pathol 2010;134(6):939-47.

[47] Tremblay A, Stather DR, Maceachern P, Khalil M, Field SK. A randomized controlled trial of standard vs endobronchial ultrasonography-guided transbronchial needle aspiration in patients with suspected sarcoidosis. Chest 2009;136(2):340-6.

[48] Zappala CJ, Desai SR, Copley SJ, Spagnolo R, Cramer D, Sen D, et al. Optimal scoring of serial change on chest radiography in sarcoidosis. Sarcoidosis Vasc Diffuse Lung Dis 2011;28(2): $130-8$.

[49] Herráez Ortega I, López González L. Thoracic sarcoidosis. Radiologia 2011;53(5):434-48.

[50] Keijsers RG, Grutters JC, Thomeer M, Du Bois RM, Van Buul MM, Lavalaye J, et al. Imaging the inflammatory activity of sarcoidosis: sensitivity and inter observer agreement of (67) Ga imaging and (18)F-FDG PET. Q J Nucl Med Mol Imaging 2011;55(1):66-71.

[51] Nunes H, Brillet PY, Valeyre D, Brauner MW, Wells AU. Imaging in sarcoidosis. Semin Respir Crit Care Med 2007;28(1):102-20.

[52] Nomura S, Funabashi N, Tsubura M, Uehara M, Shiina Y, Daimon $M$, et al. Cardiac sarcoidosis evaluated by multimodality imaging. Int J Cardiol 2011;150(2):e81-4.

[53] Londner C, Zendah I, Freynet O, Carton Z, Dion G, Nunes H, et al. Treatment of sarcoidosis. Rev Med Interne 2011;32(2):109-13.

[54] Baughman RP, Nunes $H$. Therapy for sarcoidosis: evidence-based recommendations. Expert Rev Clin Immunol 2012;8(1):95-103.

[55] Croft AP, Situnayake D, Khair O, Giovanni G, Carruthers D, Sivaguru A, et al. Refractory multisystem sarcoidosis responding to infliximab therapy. Clin Rheumatol 2012 Jan 17 [Epub ahead of print].

[56] Paramothayan NS, Lasserson TJ, Jones PW. Corticosteroids for pulmonary sarcoidosis. Cochrane Database Syst Rev 2005;(2): CD001114.

[57] Ziegenhagen MW, Rothe ME, Zissel G, Müller-Quernheim J. Exaggerated TNFalpha release of alveolar macrophages in corticosteroid resistant sarcoidosis. Sarcoidosis Vasc Diffuse Lung Dis 2002;19(3):185-90.

[58] Paramothayan S, Lasserson TJ, Walters EH. Immunosuppressive and cytotoxic therapy for pulmonary sarcoidosis. Cochrane Database Syst Rev 2006;(3):CD003536.

[59] Baughman RP, Winget DB, Lower EE. Methotrexate is steroid sparing in acute sarcoidosis: results of a double blind, randomized trial. Sarcoidosis Vasc Diffuse Lung Dis 2000;17(1):60-6.

[60] Toussirot E, Pertuiset E. TNFalpha blocking agents and sarcoidosis: an update. Rev Med Interne 2010;31(12):828-37.

[61] Milman N, Graudal N, Loft A, Mortensen J, Larsen J, Baslund B. Effect of the TNF-alpha inhibitor adalimumab in patients with recalcitrant sarcoidosis: a prospective observational study using FDG-PET. Clin Respir J 2011. doi: 10.1111/j.1752-699X.2011. 00276.x.

[62] Judson MA, Baughman RP, Thompson BW, Teirstein AS, Terrin ML, Rossman MD, et al., ACCESS Research Group. Two year prognosis of sarcoidosis: the ACCESS experience. Sarcoidosis Vasc Diffuse Lung Dis 2003;20(3):204-11.

[63] Piotrowski WJ, Kurmanowska Z, Antczak A, Marczak J, Górski P. Exhaled 8-isoprostane as a prognostic marker in sarcoidosis. A short term follow-up. BMC Pulm Med 2010;10:23.

[64] Boot RG, Hollak CE, Verhoek M, Alberts C, Jonkers RE, Aerts JM. Plasma chitotriosidase and CCL18 as surrogate markers for granulomatous macrophages in sarcoidosis. Clin Chim Acta 2010;411(1-2):31-6.

[65] Fischer T, Filimonow S, Petersein J, Zimmer C, Beyersdorff D, Guski H. Diagnosis of Heerfordt's syndrome by state-of-the-art ultrasound in combination with parotid biopsy: a case report. Eur Radiol 2002;12(1):134-7. 